\title{
Current Situation of International Marketing and Development Strategy Under the Background of Economic Globalization
}

\author{
Jing $\mathrm{He}^{1, \mathrm{a}}$ \\ ${ }^{1}$ Jiangxi Vocational Technical College of Industry \& Trade, Jiangxi, Nanchang, 330038 \\ a739960103@qq.com

\begin{abstract}
Economic globalization has intensified the competition in the international market. Chinese enterprises should innovate and develop international marketing and carry out effective international market strategic planning, which can further promote the development and development of enterprises and help to realize the development of international market.
\end{abstract}

Keywords: economic globalization, International marketing, Strategy exploration

\section{国际市场营销现状与在经济全球化背景下的发展策略 何静 $1, \mathrm{a}$}

\author{
${ }^{1}$ 江西工业贸易职业技术学院 江西 南昌 330038 \\ a739960103@qq.com
}

摘要:

经济全球化加剧了国际市场的竞争。中国企业要创新发展国际营销, 开展有效的国际市场战略规划可以进一 步促进企业的发展和发展，有助于实现国际市场的开拓。

关键词: 经济全球化; 国际市场营销; 策略探索

\section{1 市场营销概述与经济全球化特征}

\section{1 概述}

市场营销既是一种职能，又是组织为了自身及 利益相关者的利益而创造、沟通、传播和传递客户 价值，为顾客、客户、合作伙伴以及整个社会带来 经济价值的活动、过程和体系，其主要是指营销人 员针对市场开展经营活动、销售行为的过程。

市场营销的营销状况与方向在国内主要分为两 种, 一是对内的国内销售, 二是对外的出口销售。 面对经济全球化的大潮流影响, 导致目前大多数市 场营销已经开始将自身的营销范围扩大, 转变为国 际营销和国际营销和全球营销。国际市场营销的经 济贸易主体是企业, 所以企业只有在国际市场中对
产品进行销售和服务推广，才能够从中获得相应的 利益。企业在市场进行扩大的情况下, 必须去更多 的挖掘营销活动中的设计与规划工作, 提升质量才 能够更好的去开拓国际市场。

国际市场经营范围扩大会使得商品具有强大的 流通性以及商品基础上的营销活动, 只有把这些因 素牢牢把握, 才能够真正意义上打开国际市场, 这 也是国际市场营销状况的主要因素。经济全球化使 得国家与国家之间的交流加深，让企业能够更好的 在国际市场中将自身的商品进行销售, 开拓更多的 营销手段来打开国际市场, 只有这样才能够将更多 的国际贸易份额和利润掌握在企业本身手上，从而 在国际贸易市场中占据主动权。 


\section{2 经济全球化特征}

所谓经济全球化就是在全球经济发展的过程中 打破国家的地区限制, 从而实现多个国家的对外贸 易往来, 最终形成一个经济发展的共同体。经济全 球化是商品、技术、信息、服务、货币、人员、资 金、管理经验等生产要素跨国跨地区的流动, 也就 是世界经济日益成为紧密联系的一个整体。因此经 济全球化也是全球经济发展的必然, 想要真正意义 上的去把握经济全球化的主要方向, 就需要企业去 了解经济全球化主要的三个特征。

\subsection{1 经济贸易的全球化.}

随着独立国家的不断崛起, 经济的迅速高质量 发展, 全球经济逐渐开始恢复常态, 经济全球化是 当代世界经济的重要特征之一, 也是世界经济发展 的重要趋势。经济全球化的主要表现形式就是国家 与国家之间的贸易全球化这些导致出现很多自由的 多元化的贸易往来。

\subsection{2 出现大量的企业并购现象}

这种现象的出现主要是来自于经济全球化的影 响, 导致强大的跨国公司能够通过自身财力雄厚将 其他共同领域中发展较差的公司进行合并, 这种现 象出现就表示经济全球化会把国家和企业的发展进 行有机的整体结合, 使得每一个国家和企业都可以 对其他产业进行并购。

\subsection{3 科技发展带来的全球化}

科技的发展与创新是推动国家经济发展的主要 动力, 因此在经济发展全球化的今天, 国家之间的 科技逐渐开始进行交流, 使国家之间的竞争与企业 之间的竞争都开始包含科技元素，同时竞争也愈发 的激烈, 甚至已经成为影响竞争的核心要素。

所以面对这种状况就需要企业在进行国际市场 营销策略的发展过程中, 做好自身的创新意识, 提 高企业的自主创新能力, 加大对于科技研发的投入 力度, 打开国际市场中的新方向, 才能够更好的去 提升企业在国际市场中的地位。

\section{2 国际市场营销策略的现状}

\section{1 营销策略不够完善}

我国虽然长期以来一直是世界卫生组织的成员, 但如果对比很多企业的管理制度和业务流程, 仍然 可以发现和国际先进企业的差距。比如, 一些公司 的营销活动和产品不符合客户需求, 欠缺特定的国 际市场的国际营销和营销策略。由此, 公司的营销 人员需要明确营销工程形势和国际营销发展趋势, 并以此为基础, 创新及优化公司的管理方法, 由此
来促进市场营销的发展和创新。通过这种营销活动, 我们可以在国际市场上获得更多的主动权。

然而, 很多企业由于各种因素没有正确认识管 理体系优化和创新活动的重要价值和功能, 营销战 略管理显得力不从心。企业管理体系不规范也是影 响国际营销活动的因素之一。

\section{2 营销人员水平有待提高}

国内营销和国际营销的市场营销方式是有区别 的, 国际营销对营销人员的要求更高。首先, 营销 人员必须对市场有敏锐的洞察力, 熟悉不同的国外 用户的思维方式和习惯, 有一定的外语基础和与外 国客户沟通的能力, 有一定的国际视野。但是, 国 际营销人员的工作能力是不同的, 因为很多公司在 开展国际营销活动时并没有开展有关的专业课程来 培训国际营销人员, 而且国际营销人员的决策也比 较随意。部分营销人员具备外语沟通能力, 但缺乏 专业技能, 这也会对公司的国际营销发展产生一定 的负面影响。

\section{3 监督评估机制不完善}

在国际市场实施营销活动的过程中, 必须要经 过施工复杂的环节, 才能够真正的把营销活动推广 到国际市场中, 正是因为这个因素的存在, 从多个 角度来看都影响了国际市场监督和评估机制的发展, 这也正是国际市场营销活动难以开展的重要难题。 对于企业的管理人员来说，在对市场营销进行规划 的过程中, 并不能只是单方面的去对实施计划方案 进行设计, 还必须去考虑到监督与评估企业在国际 市场中营销方向。

通过调查数据分析, 可以得到目前很多企业的 管理人员, 并没有真正意义上的了解到监督工作和 评估机制, 在国际市场营销过程中的价值与地位, 这就导致企业中每一个部门在实施的过程中不能进 行有效紧密的衔接, 企业也难以在国际市场营销活 动开展的过程中保持协调性和规范性。

\section{3 经济全球化背景下的国际市场营销}

经济全球化背景下的国际市场营销策略中, 需 要重点从这几个方便出发: 面树立国际市场营销新 理念; 加强国际市场调研, 规划科学营销方案; 不 断完善企业产品策略; 加强营销人员考核; 利用现 代科技完善信息管理以及企业渠道创新策略。

其中企业渠道创新策略中要做好注重先进科学 技术; 注重数字信息化技术以及合理及时的调整销 售业务工作。 


\section{1 全面树立国际市场营销新理念}

企业在发展过程中第一要素是树立全球化的营 销理念。目前, 我们需要持续增加对经济全球化宏 观背景的认识理解, 同时企业也要综合考察自身各 方面的不足和长处, 积极参与国际营销, 在此基础 上吸纳市场人才和资源, 在这个国际经济一体化的 时代。由此实现公司的成长。

同时企业也要有文化营销的观念。现在我们已 身在知识经济全面发达的时代, 在国际市场营销时 代，企业需要将文化营销理念的应用功能和价值放 在重要的地方, 同是由文化营销的巧妙应用来吸引 更多的消费者, 引起消费者对企业产品及企业员工 服务的好奇。以上方式能够增强消费者对企业产品 的消费欲望, 由此增加公司在国际市场的影响力, 有利于提升公司在国际市场的营销能力。

最后，企业应当重视绿色营销的理念。经济发 展的同时也使得环境问题更加严重, 环境问题是各 个国家在发展期间都需要重视和解决的问题。在这 种情况下，企业在国际市场营销期间就应当坚持绿 色营销的理念, 尽可能地降低营销对环境的不利影 响。

\section{2 加强国际市场调研, 规划科学营销方案}

企业可以通过可信赖的调研活动笁选总结, 增 强对国际营销特点的认识，同时在这个基础上决定 企业的国际营销方式, 制定有效的国际营销方式和 方案, 由此, 可以保证国际营销活动的顺畅发展。 一个非凡的营销计划能够刺激营销人员的思维, 训 练营销人员的适应不同环境的能力, 这也为营销人 员的平常的活动与工作提供基础支持, 有助于避免 国际营销活动中发生的低级错误。

\section{3 不断完善企业产品策略}

国际营销的一个关键策略是产品营销策略，因 为在国际营销市场来说国际营销盈利的主要方式是 销售服务和产品。企业可以通过提高营销产品质量 进一步促进营销开展。当企业完成初步的产品改进 后，应投入充足的资金支持新产品的科学研究，优 化产品的资源配置, 最大限度地有效利用企业资源。 这样不仅能够有效地使用产品的开发技术, 同时也 能够在市场营销工作期间真正落实资源合理整合的 工作意识, 有效发挥产品的优势和价值, 确保其能 够在国际市场营销工作中更好的应用。

\section{4 加强营销人员考核}

缺乏突出的专业国际营销人才是目前中国企业 国际营销工作中最普遍的问题。目前的国际营销团 队缺乏专业性, 稳定性不高, 以及缺少合理的年龄 结构, 这在一定程度上会导致营销不利于国际营销
的发展。而培养出一支高素质、专业能力突出的营 销人才队伍, 可以有效促进企业国际营销的发展, 并且缓解国际营销的压力。

为了加强国际营销的专业基础知识和相关营销 能力, 在这种情况下, 企业必须分析国际营销的基 本情况和现有存在的问题, 并在此基础上进行相关 培训。企业也可以构建新的员工考核机制和优化营 销人员结构。

\section{5 利用现代科技完善信息管理}

科学技术的进步也不断的去影响企业的发展, 企业在顺应时代发展的过程中, 也需要去注重现代 化和信息化的发展。企业在国际市场中进行营销的 实施过程中, 就可以将科学的信息化手段运用到营 销工作中, 通过科学的管理和营销方式的创新发展, 不仅仅可以提升营销工作的工作效率, 还可以在一 定程度上减轻工作人员的压力, 同时建立健全企业 人事管理制度，从而在整体上提升国际市场营销工 作的信息化发展程度。

\section{6 企业渠道创新策略}

企业渠道作为市场营销工作的主要载体，就必 须把渠道管理工作做好扁平化才能够让企业市场营 销工作的利益最大化。

\section{6.1 注重先进科学技术}

扁平化的渠道管理工作也就证明了企业必须在 国际市场营销工作的时间范围内。更加注重先进科 学技术, 在企业产品生产过程中的运用, 不仅仅能 够提升产品的质量, 还能够加快产品在生产过程中 的生产效率, 提高产品的生产质量, 这不仅仅能够 在很大程度上对成本进行更好的把握, 也可以刺激 消费者购买产品的欲望和消费需求。

\section{6.2 注重数字信息化技术}

不仅如此, 企业在重视数字信息化技术, 在国 际市场营销中的应用，也需要去关注线下营销的发 展将线上营销和线下营销相互结合，才能够打破传 统的营销方式，在此基础上进行优化创新可以更好 的实现线上线下国际市场份额的占领，企业要想实 现国际市场营销, 还必须充分考虑到数字化分销渠 道的发展, 只有这样才能够让企业在国际市场中更 具有竞争力, 并且产品的营销渠道的丰富性, 能够 更好的去实现国际与企业的有效联系。

\section{6.3 合理及时的调整销售业务}

最后在进行国际市场营销的过程中，企业还需 要对自身与销售业务进行综合使用情况的调度, 通 过实际情况来进行策略的变通, 与时俱进, 紧跟时 
代发展的步伐，通过这样的策略来增加国际市场竞 争过程中线下线上的综合影响力。

\section{4 结论:}

在这个经济全球化的时代, 面对更加激烈的国 际市场的竞争，企业更须树立全球化营销理念，多 做市场调研，找出企业的不足优化并完善，做出科 学的营销方案, 不断随着市场的变化而优化产品的 资源配置，同时也需优秀的营销工作人员使消费者 对企业产品和服务更加了解匹配。

\section{REFERENCES}

[1] Liu Yan, Yang Nian. Research on the curriculum reform of international marketing based on the cultivation of innovation and entrepreneurship ability [J]. Journal of Jilin radio and Television University, 2019, 0 (11): 11-12

[2] Zhao Yuanyuan, He Jing. Demand analysis and Training Countermeasures of marketing talents under the background of economic globalization [J]. Journal of Guangdong agricultural, industrial and Commercial Vocational and technical college, 2019, 35 (1): 64-66

[3] Zhao Yuanyuan, Wang Yun. Integration of internationalization concept and domestic marketing education model [J]. Journal of Taiyuan City Vocational and technical college, 2018, 0 (10): $144-145$

[4] Qu Qiang, Chen Xuebo, Li Qi. Construction of curriculum system for postgraduates majoring in control engineering under the background of new engineering $[\mathrm{J}]$. Education and teaching forum, 2020, 0 (10): 247-248

[5] Wang Mengbo, Wu Zhidong, Xiao Qiaoling, Mo Huiyin. Analysis of cross-border e-commerce marketing strategy -- Taking xiaohongshu as an example [J]. Journal of Henan Radio and Television University, 2018,31 (4): 21-26

[6] Qin Zuzhi, Zong Li. Analysis on the operation strategy of traditional media advertising market -- a case study based on GXD Media Group [J]. Journal of Guangxi Normal University (PHILOSOPHY AND SOCIAL SCIENCES EDITION), 2015, 36 (4): $179-184$

[7] Zhang Jijiao. Empirical analysis on Influencing Factors of marketing innovation of "time-honored" enterprises $[\mathrm{J}]$. Journal of Guangxi Normal University (PHILOSOPHY AND SOCIAL SCIENCES EDITION), 2016, 37 (1): 6-12
[8] Wang Taosheng. Research and practice of teaching reform of international trade practice -- training of international economic and trade application talents based on Compound Innovation [J]. Journal of Changsha Railway University: Social Science Edition, 2008, 0 (3): 114-116

[9] pan Chunyu. Research on the reform and practice of international marketing teaching methods based on professional managers as resident tutors []. Journal of Tianjin Sino German University of applied technology, 2018, 0 (5): 23-26

[10] Yin Weixi's research on Bilingual Teaching Reform of economics and management courses based on the introduction model -- Taking the course of international marketing as an example [J]. Theoretical research and practice of innovation and entrepreneurship, 2019, 0 (12): 57-58

[11] Hu Lianghui. A new probe into the teaching reform of integrating Chinese excellent traditional culture into professional courses -- Taking marketing courses as an example [J]. Journal of Shaoyang University: Social Science Edition, 2020, 19 (6): 108-111

[12] LAN Jiying. Design and Research on Integrated Practical Teaching of marketing planning course under the background of "mass entrepreneurship and innovation" Education [J]. Journal of Shanxi Finance and Taxation College, 2017, 19 (1): $72-76$

[13] Wang Chunlan. Research on the construction of flipped classroom in Vocational Colleges Based on the deep integration of school enterprise Curriculum -- Taking marketing as an example $[\mathrm{J}]$. Value engineering, 2017, 36 (28): 253-256

[14] Zhu Jing. Research on the reform and practice of interactive teaching mode based on we media platform -- Taking the course of international finance as an example [J]. Modern business and industry, 2018, 39 (8): 150-152

[15] Xu Jie, Qu Weiqiang, Han Jingiian, Dong Jianwen, song Ruochen, Wang Li, Li Li, Zeng Hongyan, Feng Zhanbin, Han Lidong, Jiang Haitao, Ren Fujiao. Research on teaching reform of international economy and trade major in Higher Vocational Education [J]. Journal of Shandong Economic Management Cadre College, Shandong Institute of administration, 2005 (B05): 81-96

[16] Liu Feng. Exploration and practice of flipped classroom teaching mode based on Project-based Learning -- Taking the course of marketing as an example [J]. Higher Vocational Education: Journal of Tianjin Vocational University, 2014, 0 (6): 61-64 